\title{
A possible foodborne outbreak of hepatitis $A$ in the Netherlands, January-February 2010
}

M Petrignani (m.petrignani@ggdzhw.nl)1, L Verhoef², R van Hunen'1, C Swaan³, J van Steenbergen³, I Boxman4, H J Ober5, H Vennema ${ }^{2}$, M Koopmans ${ }^{2}$

1. Department of Infectious Disease Control, Public Health Service, Zoetermeer, the Netherlands

2. Laboratory for Infectious Diseases and Screening, Centre for Infectious Disease Control, National Institute for Public Health and the Environment (Rijksinstituut voor Volksgezondheid en Milieu, RIVM), Bilthoven, the Netherlands

3. Preparedness and Response Unit, Centre for Infectious Disease Control, National Institute for Public Health and the

Environment (Rijksinstituut voor Volksgezondheid en Milieu, RIVM), Bilthoven, the Netherlands

4. Food and Consumer Product Safety Authority, Zutphen, The Netherlands

5. Food and Consumer Product Safety Authority, Amsterdam, the Netherlands

Citation style for this article:

Citation style for this article: Petrignani M, Verhoef L, van Hunen R, Swaan C, van Steenbergen J, Boxman I, Ober HJ, Vennema H, Koopmans M. A possible foodborne outbreak of hepatitis A in the Netherlands, January-February 2010. Euro Surveill. 2010;15(11):pii=19512. Available online: http://www.eurosurveillance.org/

ViewArticle.aspx?Articleld $=19512$

This article has been published on 18 March 2010

As of 1 March 2010, a total of 11 primary cases with onset of symptoms between 31 December 2009 and 10 February 2010, have been identified with identical hepatitis A genotype IB strains in the Netherlands. A relation with Australian and French foodborne outbreaks occurring in 2009 and 2010 is suspected. Ten of the 11 primary cases indicated that they had consumed one or more products containing semi-dried tomatoes during their incubation period.

On 12 February 2010, the virology reference laboratory for hepatitis A sequencing in the Netherlands detected a new hepatitis A virus (HAV) strain in five patients with acute hepatitis. The patients did not reveal common exposures and they were geographically dispersed. Their onset of disease ranged between 11 and 22 January 2010. Although the number of reported cases was normal for the time of the year, finding five identical HAV genotype IB strains was unusual and led to an outbreak investigation that is still ongoing in the Netherlands. Here we describe the preliminary results of this ongoing investigation.

\section{Epidemiological investigation}

The cases included in the cluster were defined as all reported hepatitis $A$ infections in the Netherlands with date of onset of disease from 15 December 2009 until present, with viruses with an identical sequence in a fragment of the $\mathrm{VP}_{1-2} \mathrm{~A}$ region $[1,2]$.

The cases included for a case control study were defined as all reported hepatitis $A$ infections in the Netherlands with date of onset of disease from 15 December 2009 until present. Exclusion criteria were:

- most probable source of infection outside the Netherlands or outside any western European country,
- most probable route of transmission sexual contact between men,

- detection of a non-related HAV strain,

- secondary cases.

The absolute number of reported cases in the period under investigation, January and February 2010, was 39 and the proportion of cases that contracted their infection in the Netherlands was $82 \%$. This number is not elevated compared with previous years. Between 2005 and 2009, the number of HAV reports in the Netherlands in January and February had ranged between 23 and 44 , with a median of 33 . The proportion of cases that contracted their infection in the Netherlands in these months ranged between $66 \%$ and $80 \%$, with a median of $68 \%$, and mostly reflects onward transmissions following the wave of travel-associated primary cases that is usually seen in autumn $[3,4]$.

Of the 39 cases notified in January and February 2010 (Figure 1), 24 had no history of recent travel abroad, denied sexual contact between men and had no known relation to another patient or cluster.

Serum samples from 31 of the 39 notified persons were available for PCR. Of these, 21 yielded a PCR product that could be used for sequencing. The genotypes identified were IA (three patients), IIIA (two patients) and IB (16 patients). Of the 16 IB sequences, 13 were identical with closest genetic relatedness to viruses identified in travellers returning from Turkey, and three were distinct and clustered with strains commonly identified in travellers from Morocco. The 13 patients with identical strains were contacted for further investigation.

As of 1 March 2010, a total of 11 primary cases, six male and five female aged between 20 and 63 years, with onset of symptoms between 31 December 2009 and 10 February 2010, have been identified with identical HAV 
genotype $1 \mathrm{~B}$ strains. Ten of the 11 primary cases indicated that they had consumed one or more products containing semi-dried tomatoes during their incubation period. The 11th case could not be reached. Two additional cases infected with the same strain are considered to be secondary cases (Figure 1). Both were closely related to a primary case and their onset of symptoms was approximately two weeks after the onset date of the suspected index case.

Two male patients in their late 30 s and 50 developed liver failure, for which they needed a liver transplantation. They did not have underlying liver disease. We are unable to explain the severe outcome of these two patients. Usually, the rate of fulminant liver disease is less than $1,5 \%$ of hospitalised hepatitis A patients [5].

\section{Related outbreaks}

The HAV strain was found to be identical to an HAV IB strain involved in food-related hepatitis A outbreaks in Australia during 2009, based on a $300 \mathrm{nt}$ overlapping sequence of the VP1-2A part of the genome (kindly provided by MJ Lyon, Public Health Virology Laboratory, Queensland, Australia) [6,7]. Furthermore, an outbreak of hepatitis A had occurred in France between November 2009 and January 2010 (personal communication). The strain identified in the French outbreak (kindly provided by AM Roque-Afonso, Laboratoire de Virologie, Hôpital Paul Brousse, Villejuif, France) also belonged to the IB genotype, but differed in $2 \mathrm{nt}$ from the Australian strain (based on a $300 \mathrm{nt}$ fragment), and in $3 \mathrm{nt}$ from the Dutch strain (based on a $430 \mathrm{nt}$ fragment).

Although this is a small difference, it should be considered significant, as typically a single unique strain is observed in outbreaks of HAV. Having said that, both strains cluster with viruses known to circulate in the same geographic region that includes Turkey. This is concluded on the basis of sequence data obtained from HAV-infected returning travellers. It does not provide robust evidence for a source of infection, because the level of sampling in populations in the wider region is insufficient.

\section{Source tracing}

Since no other epidemiological connection between the cases could be made, a common food source was considered most likely. A case control study was initiated to assist in identifying the food product involved, and results are not yet available.

In case control studies in Australia and France, the recent occurrence of $\mathrm{HAV}$ infection was associated with consumption of semi-dried tomatoes. Therefore, the Dutch Food and Consumer Product Safety Authority started an investigation focusing on products containing this ingredient eaten by the primary cases in the current outbreak. These differed in the way they were presented for purchase and were purchased in different supermarkets, markets or delicacy stores. Full trace back to the area of production is ongoing. So far, ten different product types of semi-dried tomatoes have been identified as consumed by the Dutch cases, imported from three different countries. No original samples are available for investigation, but as yet, 52 food samples of similar products have been tested, in which HAV RNA could not be detected. No common producer or distributor could be identified so far that would explain all the Dutch cases.

The same applies for a link between the outbreaks in the Netherlands, Australia and France. France was able to trace the batch of semi-dried tomatoes implicated in the French outbreak, but no leftovers of this specific batch were found. Because the French and Dutch/ Australian HAV strains were not identical, the exact

\section{FIGURE}

Cases of hepatitis A notified in the Netherlands in January and February 2010 ( $n=39)$

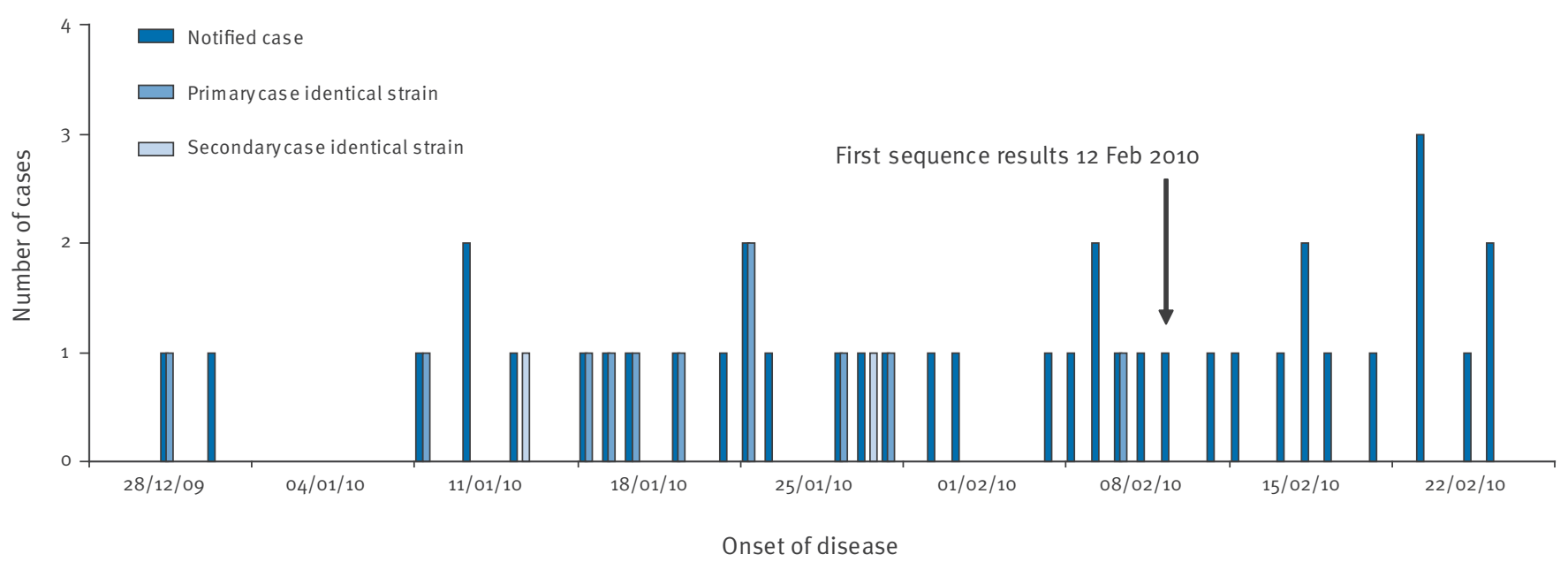

Notified cases include all notifications in this period. Primary and secondary cases include those cases with an identical strain related to the possible food-borne cluster, identified as of 1 March 2010. 
sources and modes of transmission of the outbreak in the Netherlands remain to be established.

\section{Conclusions}

We have identified a cluster of patients infected with an identical HAV IB strain. As the partial strain sequence showed a $100 \%$ match with viruses found as the cause of foodborne outbreaks in Australia, and high similarity with the HAV strain causing a recent foodborne outbreak in France, a possible common source to these outbreaks is currently being investigated. Trace back investigations so far showed a highly complex market for one of the products considered as a possible source (semi-dried tomatoes), and failed to identify a common link between all cases. This is similar to observations in Australia where after an initial small outbreak, a second wave was observed that involved a large increase in locally-acquired cases compared to previous years [6,7 and personal communication]. Therefore, although we have not received reports of confirmed primary cases since 17 Feb 2010 (onset of disease 10 Feb 2010), this calls for vigilance in the weeks to come.

We are interested in all cases that may be linked to this outbreak. Strains can be compared using the HAV database of the Food-borne Viruses in Europe (FBVE) network at the Dutch National Institute for Public Health and the Environment (Rijksinstituut voor Volksgezondheid en Milieu, RIVM). For details, please contact fbve@rivm.nl.

References

1. Grinde $B$, Stene-Johansen $K$, Sharma $B$, Hoel $T$, Jensenius $M$, Skaug K. Characterisation of an epidemic of hepatitis $A$ virus involving intravenous drug abusers--infection by needle sharing? J Med Virol. 1997;53(1):69-75.

2. Stene-Johansen K, Tjon G, Schreier E, Bremer V, Bruisten S, Ngui SL, et al, Molecular epidemiological studies show that hepatitis $A$ virus is endemic among active homosexual men in Europe. J Med Virol. 2007;79(4):356-65.

3. van Steenbergen JE, Tjon G, van den Hoek A, Koek A, Coutinho RA, Bruisten SM. Two years' prospective collection of molecular and epidemiological data shows limited spread of hepatitis A virus outside risk groups in Amsterdam, 20002002. J Infect Dis. 2004;189(3):471-82.

4. van Gorkom I, Leentvaar Kuijpers A, Kool JA, Coutinho RA. [Association between the yearly hepatitis A epidemic and travel behaviour of immigrants in the four major cities of the Netherlands]. Ned Tijdschr Geneesk 1998;142(34):1919-23. [Dutch]

5. Centre for Infectious Disease Control, RIVM Bilthoven. The Netherlands. [Internet]. [Hepatitis A guideline]. May 2009. Available from: http://www.rivm.nl/cib/infectieziekten-A-Z/ infectieziekten/HepatitisA/. [Dutch].

6. Hepatitis A virus, semi-dried tomatoes - Australia: recall. ProMED-mail [online]. Boston US: International Society for Infectious Diseases; 22 May 2009. Archive no. 20090522.1917. Available from: http://www.promedmail.org.

7. Hepatitis A virus, semi-dried tomatoes - Australia (03):

(Victoria). ProMED-mail [online]. Boston US: International Society for Infectious Diseases; 4 November 2009. Archive no. 20091104.3811. Available from: http://www.promedmail.org. 